\title{
GIS Based Integration of the Health Emergency Services Regional Network in Morocco
}

\author{
Ibtissam Khalfaoui, and Amar Hammouche
}

\begin{abstract}
The aim of this research is to improve the Moroccan Health Emergency Services (HESs) at the regional level. To do that, the HESs-RN (Regional Network) Geographic Information System (GIS) mapping was established on the basis of 1) proposing the structuring of HESs in the form of the HESs-RN modeled as a graph 2) modeling the HESs-RN by considering a medical emergency event as the Occurrence of events of Health Emergency (OHE), 3) proposing a Decision Support Model (DSM) to manage and control at best the different HESs-RN's OHE, and in particular to determine, in real time, the fastest path for the patient's transfer. This DSM supposes that the HES-RN nodes cooperate autonomously using best practices HES protocols and that they are regulated by the Health Emergency Assistance node.
\end{abstract}

Index Terms-Ambulance routing, decision support model, health regional network modelling, GIS mapping.

\section{INTRODUCTION}

As expressed by [1], health is a prerequisite for all mankind. It is a critical component of individuals' social needs. A health system is defined as a set of elements that determine the health status of a population. The Moroccan Healthcare system faces multiple challenges in improving and ensuring the health and wellbeing of the Moroccan citizens, especially at the medical emergency level.

Indeed, the effective management of emergency services has become a critical issue in the hospital sector in Morocco. It is well known that these services are often congested, under-equipped and suffering from a degraded image with performance levels below those set by international standards.

To bring solutions of improvement of the organization and the functioning of such services at a territorial region level, their structuring and management are considered as part of the HES-RN of RSZZ (Rabat, Sale, Zemmour ${ }^{1}$, Zaer).

This study allows, among other things, to provide a gateway linking and integrating all HESs-RN nodes with each other in a graph, by means of a GIS mapping allowing communication, coordination, transparency and data sharing, with the aim of increasing patient satisfaction.

The main contributions of this paper are:

- Structuring of 1) a database, in the form of a Geographic Information System (GIS) mapping of the HES-RN of RSZZ (Rabat, Sale, Zemmour, Zaer in Morocco), and integrating sets of information, necessary to manage and to well regulate the network, and of 2) the patient's data

Manuscript received February 11, 2019; revised June 6, 2019. This work was supported in Mohammadia School of Engineers, Morocco.

The authors are with Mohammadia School of Engineers, Morocco (e-mail: khalfaoui.ibtissam.gip@gmail.com,amar@emi.ac.ma). related to its $\mathrm{OHE}$.

- Modeling the HES-RN-RSZZ in the form of a graph $G(V$, $E, w)$ using a GIS Software, where $V$ are vertices (Care Units - CUs), $E$ are edges (roads or communication links), and $\mathrm{w}$ are weights.

- Proposing an adaptation of an efficient routing algorithm that finds the best path between the OHE location and the Care Unit of destination (CUd), taking into consideration the distance and the flow of that path as well as the patient's condition.

- $\quad$ Proposing a Decision Support Model (DSM): the GIS mapping is the basis of this DSM to manage and pilot at best the various OHEs of the HES-RN.

\section{LITERATURE REVIEW}

In terms of managing healthcare institutions, [2] proposed a cloud-based portal and a web-service API in order to access and manage shared data.

The authors [2] introduced a framework that characterizes the essential elements of a system that provides a shared common view of the essential data for a person to any healthcare provider involved in the circle of care: 1) minimal data set, which is composed of patient profile, medications, health profile, and appointments, 2) information technology architecture (myPHR website), 3) and legal governance. The proposed framework enables patients not only to access the MyPHR website but also to update their profiles, as well as their past healthcare encounters.

Other researches [3] and [4], dealing with problems in health sector have been carried out using GIS. The tool can be used by health planners to make informed decisions about interventions to increase access to emergency services. The researchers defined GIS as a helpful tool when describing referral networks and identifying which facilities to prioritize for upgrading, and finally how these strategies impact the population's access to emergency services.

According to [5] and [6], Healthcare information system promises to optimize the workflow [5], increase legibility, reduce medical errors, shrink costs and boost the overall quality of healthcare [6]. In this context, [7] proposed a model where all information from healthcare providers (hospitals, clinics, emergency rooms, small offices, multispecialty groups, etc.) are entered into an electronic health record. This information is then networked to regional and national databases through electronic exchange. Data flows from EHRs (Electronic Health Record) and regional registries are then channeled into standards for prevention and treatment, which can be further processed to yield information 
for decision-making and decision-support.

\section{Methodology}

This study is interested in modeling the HES-RN-RSZZ structure and dynamics with a GIS software in order to optimize its functioning (see figures below).

Using a GIS model, the studied network is represented by a graph. This later is composed by a finished number of vertices (Health institutions), which are connected by edges and characterized by events, OHEs.

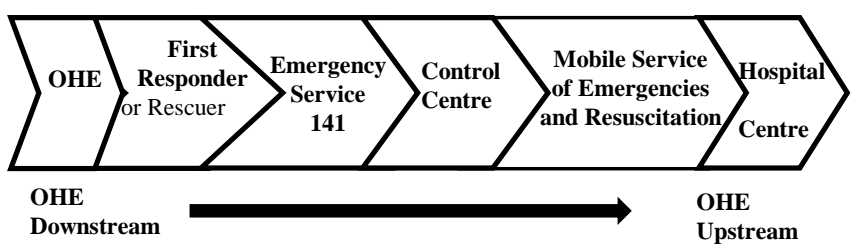

Fig. 1. OHE process.

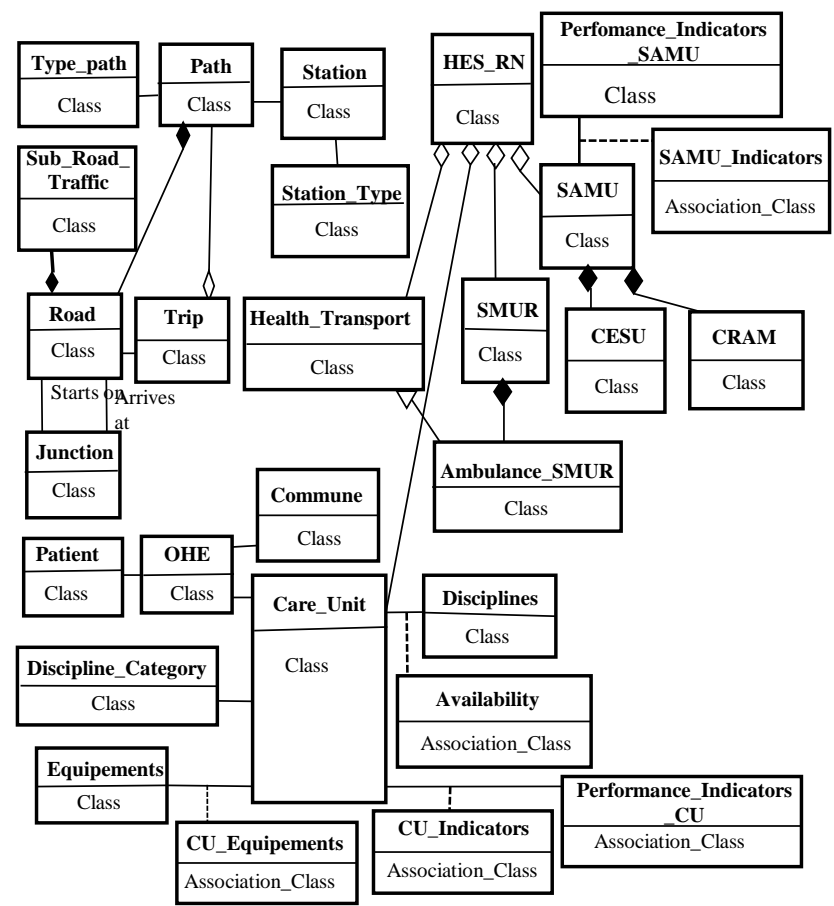

Fig. 2. HES-RN class diagram.

\section{A. HES-RN Structure Modeling}

The first step in modeling the HES-RN structure, required data collection form several organizations (Hospitals and Ambulatory Care Direction, Regional Health office, Health Delegations of RSZZ region...) and from QGIS Open source database. Once the data were gathered, the HES-RN's mapping was developed from the digital map of the studied region, under a GIS platform.

The network's mapping created was accomplished in several layers, each one having its own data, necessary to manage and regulate well such a network. It includes:

- A map of the studied region

- All data concerning the care offer (the unit name, the category, the province, the environment, the litter capacity, number of available beds, specialties, Medical staff, paramedical staff, equipment, state of the equipment).
- Patients Dossier containing multiple information about the OHE history.

- Information about existence, number and state of means of transportation (ambulance, mobile care unit, helicopter) of the region's care units.

- $\quad$ Streets and avenues of the cities of Rabat, Sale, Temara and Khemisset, with their names, and the drawing of inter-cities / villages roads.

- Train and tram's rails of the studied region.

- Drawing of tunnels and bridges.

- Information concerning public transport of the region RSZZ.

- Information concerning traffic lights, turning points and stops.

- Real time traffic conditions of the studied region.

- Road constraints represented by some schools and universities.

\section{B. HES-RN Functioning Modeling}

Studying the network's functioning or dynamics consists on studying the different OHE events, using the GIS mapping database (see Fig. 3, Fig. 4 and Fig. 5):

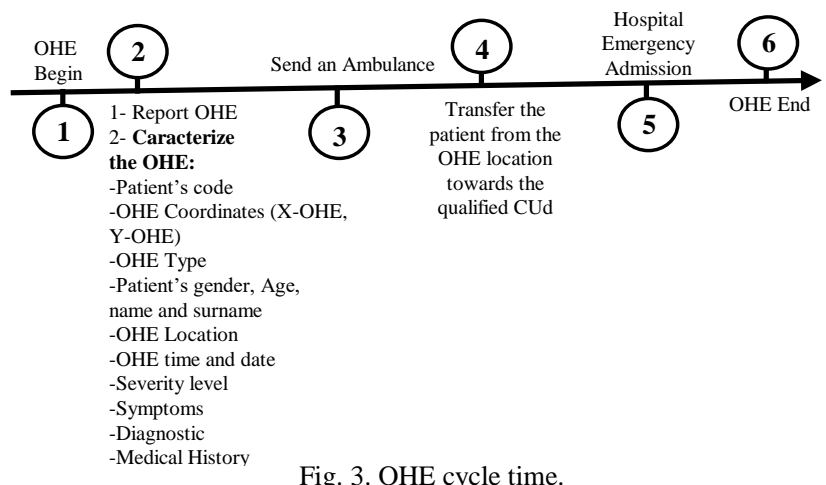

F. 3. OHE cycle time.

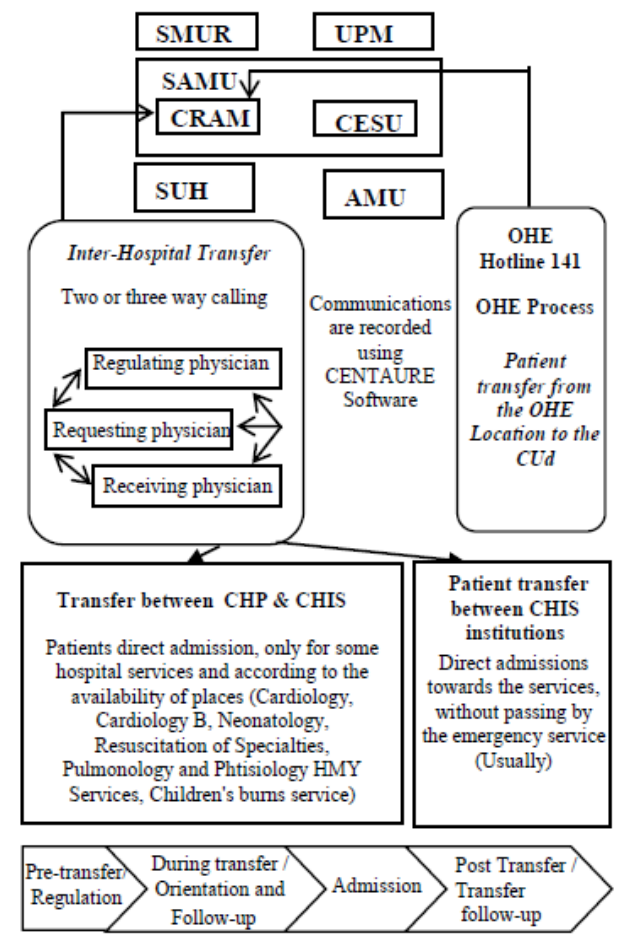

Fig. 4. HES-RN functioning.

The dynamic routing of the patient concerned by the OHE 
depends on the length of the considered road and also on the traffic flow along this road. This flow can be characterized by the road density varying in time and in space. The GIS that is used has a tool, which allows to determine, in a given radius, the CUs of destination and the shortest path which lead there. Yet, in the decision support model used, in order to transfer the patient as quickly as possible to the CUd, it is necessary to find out, in quasi-real time, the fastest path between the OHE and the CUd.

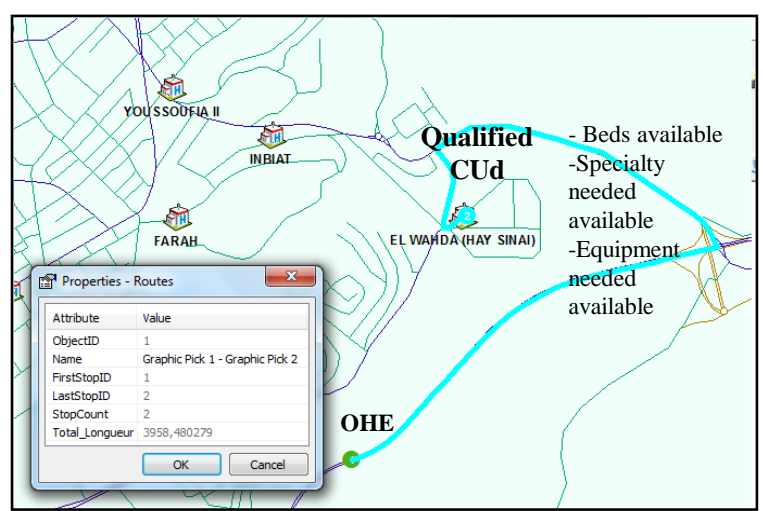

Fig. 5. OHE representation inside a road network.

In order to model the network in all its aspects, Unified Modeling Language (UML) was used. This allowed to model 1) the structure of the network as UML classes, 2) the use of the network as use cases diagram 3) the dynamic aspects of the network through a) the activity diagram that represents the behavior of a use case, and b) the sequence diagram which describes how the elements of the system interact with each other and with the actors interacting with network.

\section{1) Unique patient dossier}

The quality of care is evidence-based, patient-centric, timely, and safe. The key to evidence-based care is to be able to share previous experiments and experiences; successful or failed. As mentioned before, the biggest hole in the current healthcare practice is the absence of or inefficiencies in sharing data inter-organizationally [1].

Indeed, the traceability is a strategic asset for decision support management. In the absence of an effective traceability system, it is not possible to monitor and trace patient flows, nor to objectively measure the effectiveness of the activities of medical staff.

Too often, time is wasted repeating collection of information in an ad hoc manner, and too often critical information is missed or provided too late [1]. To this effect, a patient Dossier was created. This Dossier contains all the important information about the concerned patient.

It is therefore a matter of codifying each of the OHEs processed, of collecting all the information relating to it, of archiving the OHE Dossier, and of consulting the history of the Dossier whenever it is necessary. Moreover, this later allows to visualize, analyze, interpret and display the OHE medical and geo-location data.

\section{2) Patient transfer}

The problem of finding the fastest path to ensure a transfer from point $A$ to point $B$ of a road network is well known. Its formulation remains simplified and its resolution is complex especially at the mathematical level [8] and [9]. Even the exact solutions developed for the simplified models provide only local optimality.

Furthermore, the stochastic nature of the road traffic only complicates things. In the absence of having an optimal global solution for this problem, we settle for a meta-heuristic that allows us to provide a good approximate solution. For this, the layer "Real-Time Traffic Conditions" was built and added to our HES-RN's cartography, to have quantification and meta-visualization of road traffic at quasi-real time in this HES-RN (see Fig. 6)

There are four levels of traffic quantification adopted by ArcGIS Online. These levels are qualified as follow: [stop and go] for the densest (average minimum speed: $v_{\text {min }}$ ), [slow] with average speed $v_{1}$, [medium] with average speed $v_{2}$, and [fluid] with maximum speed $v_{\max }$.

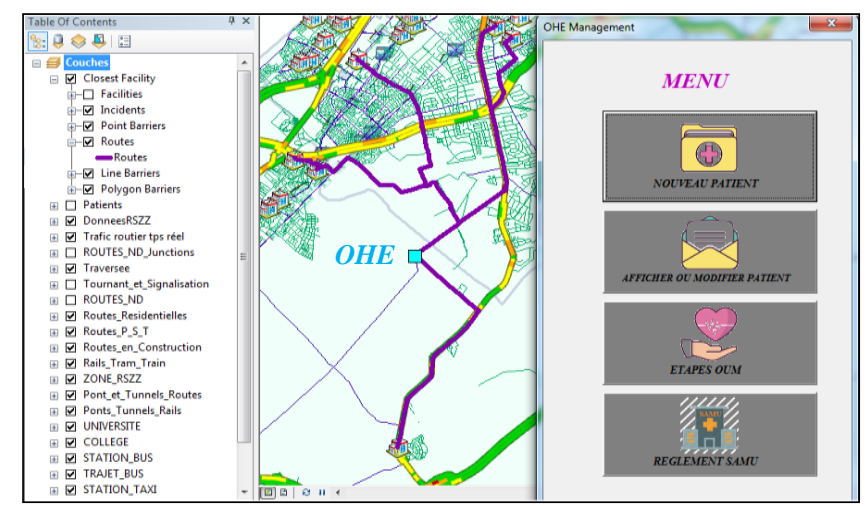

Fig. 6. HES-RN GIS mapping with near-real time meta-traffic.

Thus, on the basis of this information and the GIS data of the modelled HES-RN-RSZZ, the following algorithm is proposed, to obtain the fastest possible transfer of the patient between the OHE and the CU of destination:

1. Locate the OHE location (geographical coordinates) and characterize it.

2. Determine which CUs of destination are qualified (feasible points) to process the OHE. (See Fig. 7)

3. Classify these qualified CUs of destination (depending on OHE severity) according to their qualifications.

4. Determine all paths connecting OHE location to the qualified and classified CUs of destination, within a given radius (this latter is determined on the OHE severity).

5. Among these paths, determine the path that optimizes the transfer time of the patient according to its length and its traffic:

a. Insert in a table, all the segments of the paths found.

b. Characterize and classify these edges <edge_id, Time, Condition, average speed $>$.

The steps in this procedural heuristic that have some complexity are steps 2,4 and 5 . The difficulty for step 2 is to determine the qualification elements of a destination US and to qualify the severity levels of the OHE.

As for step 4, the problem faced concerns the graph theory, which consists in finding all the possible connection paths between two given nodes of a graph. In our case, these nodes are the OHE location and the chosen CU of destination. In this paper, several paths linking two nodes of the network, in a given radius, will be determined in order to simplify the algorithm and reduce its time of simulation. 
As for step 5, its complexity is to find, dynamically, the shortest path (fastest in our case) between two nodes. In our research, the focus is kept on these three steps.

In this study, a path is composed of a finite number of sections (edges). Every section is characterized by:

$<$ edge_id, Time, Condition, average speed $>$.

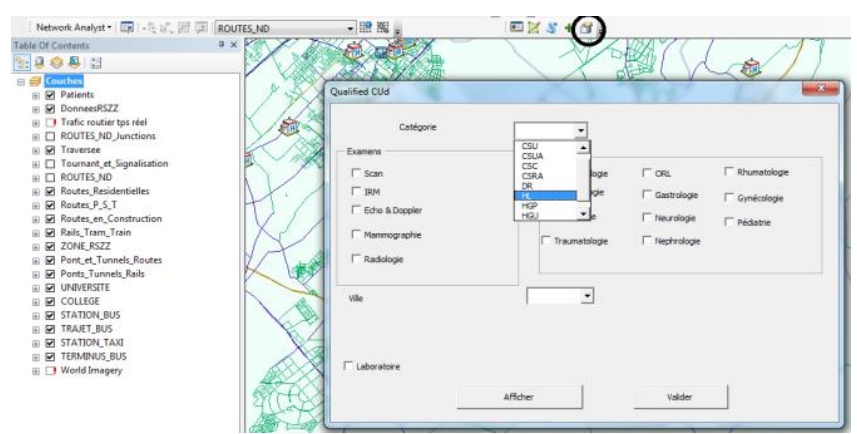

Fig.7. Query for qualified Care Units of destination.

Let $\mathrm{Vg}$ be the average speed of the path. Then steps 2, 3 and 4 become (see Fig. 7):

- Query the HES-RN database, in order to determine all CUs (qualified CUs) of destination with the necessary and the sufficient Hospital Emergency Processes (HEP) to treat the $\mathrm{OHE}$, knowing that the corresponding $\mathrm{P}-\mathrm{OHE}$ is described by its qualified UML Diagrams.

Let $\mathrm{S}$ be the non-empty set of these qualified $\mathrm{CU}$, with $S=\{\mathrm{CUi} / i=1, n\}$.

- Classify $S$ according to the CUi qualification/availability and the OHE severity. Let $S^{*}$ be this classified new set.

- Find several paths connecting the OHE location to these qualified CUs.

Set $\mathrm{R}_{\mathrm{i}}=\left\{r_{i k} / k=1 \ldots m\right\}$ the non-empty set of paths connecting the $\mathrm{OHE}$ location to the $\mathrm{CUi}$, such as:

$t_{i k}=$ travel time of the path $r_{i k}=$ (Total length of path with fluid flow) $/ V_{\max }+$ (Total length of path with slow flow) $/ V_{1}+$ (Total length of path with medium flow) $/ V_{2}+$ (Total length of path with stop and go flow) $/ V \min$.

Set tiki $=\min \{t i k, k=1 \ldots m\}$ for $k=k i$.

Then $r_{i k i} \in R_{i}$, is the fastest path between the OHE location and the CUi, and $t_{i k i}$ is its travel time.

- Classify CUi in ascending order of $t_{i k i}$, in a new list $U^{*}$.

- $\quad$ The CU, ranked first in $U^{*}$ and denoted CU*, member of $S^{*}$, is the CU of destination for this OHE.

On the other hand, and considering the dynamic of traffic road in real time, a dynamic optimization by section method is adopted: at iteration $i$ and at the current node, the objective is to find the next connected node on the optimal path of the remaining network.

In the general case of the road network, modeled as a non-oriented graph with circuits, reaching the destination node (the CUd in our case) is not guaranteed. Therefore, the assumption that the nodes cannot be revisited has been made. In this case, our problem is formulated as a Discrete Dynamic One to Some Shortest Paths (D2OS2P) problem. Dynamic, because in our modeling the system state change is considered, particularly road travel times and availability change of the CUd in time. Discrete, because changes in the system states, will be considered only at the next node on the traveling path.

Our dynamic optimization method for this D2OS2P becomes:

- Iteration 0: initial graph $G 0=$ Road network between the OHE location (Source node) and the CUd (destination node).

New initial node $=$ next connected node on the optimal path of $G 0$

New remaining road network $G 1=G 0-$ \{ Source node of $G 0\}$ - $\{$ arcs connected to the source node of $G 0\}$

- Iteration i: Initial graph $G i=$ Road network between the initial node of iteration $i$ and the CUd, with eventually new weights (travel times), and /or new eventual CUd ranking.

New initial node $=$ Next connected node, located on the optimal path of $G i$.

New remaining road network $G i+1=G i-\{$ initial node of $G i\}$ - $\{$ edges connected to the initial node of $G i\}$.

- $\quad$ Repeat iteration $i$ until the new initial node is the CUd. As an example of application of this method, see Fig. 8.

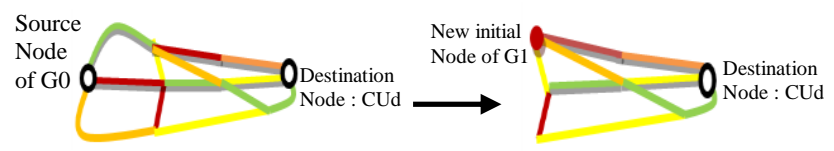

Fig. 8. Iteration 0 and iteration 1.

To implement this algorithm it is needed, at the next node 1) to determine the new travel times following any perturbations in sub-graph Gi, and/or the new eventual CUd ranking, and 2) to propose an algorithm to find the optimal path of $\mathrm{Gi}$.

Concerning point $\mathrm{n}^{\circ} 1$, it is known that the segment travel times is a function of time, speed and traffic flow.

Finding a real time solution for it, necessitates the resolution of an ill-formed problem that involves differential equations. A lot of research has been done [8] and [9] to solve this hard problem. But only approximate and incomplete solutions have been proposed. Therefore, and for practical reasons the averaging method by segment is adopted to be able to compute an average segment travel time as a function of its average travel speed. For this the following car speeds have been retained [10]:

- $\quad$ Regime I: the car speed is 48 kilometers per hour (kph) in the city streets and $65 \mathrm{kph}$ in the suburban area.

- $\quad$ Regime II: the car speed is $39 \mathrm{kph}$ in the city streets and $51 \mathrm{kph}$ in the suburban area.

- $\quad$ Regime III: the car speed is $24 \mathrm{kph}$ in the city streets and $32 \mathrm{kph}$ in the suburban area.

- Jam point [stop and go]: the car speed is between $13 \mathrm{kph}$ and 0 and the density is 0.12 vehicle for meter for lane.

As for the ranking of the different CUd, any change in this ranking will require an update of $S^{*}$.

Concerning point $\mathrm{n}^{\circ} 2$, we resorted to an adaptation of the algorithms FG proposed by Faro and Giordano [10], which deals with the APSP problem, considered as a generalization of our case study (D2OS2P).

They are used to determine 1) the Shortest Path (SP) of the considered APSP, in case the travel time of a given road changes $\left.\left(\mathrm{FG}_{\mathrm{A}}\right), 2\right)$ to find alternative shortest paths both in free flow regimes $\left(\mathrm{FG}_{\mathrm{B}}\right)$ and 3 ) in congested flow regimes $\left(\mathrm{FG}_{\mathrm{C}}\right)$.

In our case, and in order to find the new $U^{*}$, at the next 
node, an adaptation of these algorithms to the D2OS2P, is in the making.

\section{KEY PERFORMANCE INDICATORS AND DECISION SUPPORT MODEL}

In order to analyze and evaluate the activity of healthcare institutions and to respond to the needs of decision makers, within the network, a pilot dashboard of Key Performance Indicators (KPI) is used. It is a tool of control, diagnostic, dialogue and communication.

There are two types of KPI, 1) pre-hospital KPI, such as: calls waiting time, Response time, vehicle kilometers travelled, number of calls processed, average call processing time, survival rate during transfer, transfer time, number of vehicles used per day, number of transfers performed per day, and 2) Intra-hospital KPI, such as: number of urgent consultation, number of expected outpatient surgeries, number of surgical interventions scheduled, number of day hospital activities, number of examinations (scanner, Echo \& Doppler...), number of explorations, full hospitalization, Day Hospital, Average Occupancy Rate, average duration of stay , turnover rate, mortality rate, number of deliveries per year, number of medical consultations per year, total admission, emergency room, etc.

The current absence of pre hospital indicators is considered as a gap in the information available in respect of the level and quality of services in Morocco.

To address that problem it is proposed to record the OHEs data including road traffic historical data, as a layer of the GIS mapping. The information stored will be used in order to compute vehicles distances travelled, transfer times, number of vehicles used per day, number of transfers performed per day, as shown in the table below. This will allow as to evaluate the networks performance, in particular the efficiency of the Medical call control center (CRAM) and the Mobile emergency and resuscitation service (SMUR).

As to the other pre hospital KPI, they can be calculated using OHEs database of the created mapping and the Centaure Software. This later is used by the CRAM in order to record Emergency calls, the time and the duration of each call.

TABLE I: ROAD TRAFFIC TRACE (EXAMPLE)

\begin{tabular}{clll}
\hline \hline Date \& Time & OHE_id & $\begin{array}{l}\text { Ambulance } \\
\text { _id }\end{array}$ & $\begin{array}{l}\text { Path [(edge_id, start_time, } \\
\text { end_time })\end{array}$ \\
\hline Timestamp 1 & 1 & A1 & $\begin{array}{l}(\mathrm{e} 1,9,15)(\mathrm{e} 2,15,25) \\
25,31)\end{array}$ \\
\hline Timestamp 2 & 2 & A1 & $\begin{array}{l}(\mathrm{e} 2,15,19)(\mathrm{e} 3,19,23) \\
(\mathrm{e} 4,23,30)(\mathrm{e} 7,30,33)\end{array}$ \\
\hline Timestamp 3 & 3 & A3 & $(\mathrm{e} 4,22,29)(\mathrm{e} 5,29,31)$ \\
\hline$\ldots . .$. & $\ldots$ & $\ldots$ & $\ldots . .$. \\
\hline
\end{tabular}

Furthermore, to achieve optimal decision-making and to act quickly and efficiently in the emergency sector, when there is an OHE, a decision support model is proposed, using:

- $\quad$ A HES-RN GIS mapping able to provide real-time data and information needed to determine the most appropriate Care Unit (CU) of the HES-RN to receive the patient concerned with the $\mathrm{OHE}$, and to select the fastest routing for the transfer of this latter, taking into account the possible routing times to the unit care of destination.

- An algorithm allowing to determine the fastest routing of the patient, in real time, according to the candidate routings, their lengths and their traffic flows.

- A global modelling of the OHEs within the HES-RN, using UML diagrams, allowing to represent the functioning of the network in a precise and complete way.

This decision support model assumes that the HES-RN nodes work autonomously with provided regulation by the Control Centre node, insuring the access to the most effective care in the shortest possible time, with a sustainable quality of service, level of service and the best possible and with the least possible costs.

\section{CONCLUSION AND PERSPECTIVES}

This paper has presented a proposal for a reference model of a HES-RN organization and management.

The research provides with a new perspective and a framework of assisting the regional medical emergency control center to respond when the OHEs takes-place before ensuring the patient's safe and fast transfer. Our study is unique in terms of proposing an algorithmic decision procedure for the optimization of OHE Cycle Time in particular and of the critical decision processes through the HES-RN-RSZZ network in general.

By using UML to model the network's structuring (class diagram) and functioning (Activity and sequence diagrams), the current situation of the network has been analyzed and then the obstacles and challenges facing a regional Moroccan health system at the pre-hospital level have been identified. This addressed the first research objective of this study.

Then a GIS mapping was proposed to show the geographical distribution of the CUs and their description in detail.

Moreover and using this mapping, the management of OHEs, through the HES-RN, has been programmatically implemented. This addressed the second research objective of this study.

Finally, an algorithmic decision procedure has been proposed that deals with D2OS2P problem, in order to obtain the fastest possible transfer of the patient, between the OHE and the $\mathrm{CU}$ of destination and taking into account road congestions and the consequent flow perturbation that propagates over the network.

At present, the work on the implementation and the simulation of this algorithmic decision procedure is being carried out on the ArcGIS platform.

What does remain to be considered as a main perspective for the future is the study of the integration of the different Moroccan HES-RNs into a National Health Emergency Network of Morocco. With the hope that this new framework would facilitate and promote the sharing of best practices and enhance communication among the health care practitioners, and hence would allow for the significant improvement of the level and the quality of service for the patients concerned by the OHEs. 


\section{APPENDIX. LIST OF ACRONYMS}

In this paper some acronyms are used such as 1) HES-RN (Health Emergency Services Regional Network), 2) OHE (Occurrence of events of Health Emergency), 3) SAMU (Medical Emergency Assistance (Control) Centre), 4) CRAM (Medical call control center), 5) CESU (Emergency care teaching center), 6) SMUR (Mobile emergency and resuscitation service, whose activity is coordinated by the CRAM of the regional SAMU to which they are attached), 7) AMU (Means of transportation mobilized by the CRAM within the framework of the emergency medical help), 8) SUH (Hospital Emergency Services), 9) UMP (primary health-care facility, providing local medical emergency services), 10) CHIS (Ibn Sina University Health Centre), 11) CHP (Provincial state hospital) and 12) DSM (Decision Support Model).

\section{ACKNOWLEDGMENTS}

This work is part of the research project $\mathrm{N}^{\circ}$ SIT05 / 09, funded by Mohammed V University, within the framework of the agreement between this University and Ibn Sina Hospital Center.

\section{REFERENCES}

[1] A. I. Ramzi and M. A. El-Bedawi, "Towards integration of remote sensing and GIS to manage primary health care centers," Applied Computing and Informatics, 2017

[2] M. Azarm, C. Backman, C. Kuziemsky, and L. Peyton, "Breaking the Healthcare Interoperability Barrier by Empowering and Engaging Actors in the Healthcare System," in Proc. the 7th International Conference on Current and Future Trends of Information and Communication Technologies in Healthcare, 2017, vol. 113, pp. 326-333.

[3] P. E. Bailey, E. B. Keyes, C. Parker, M. Abdullah, H. Kebede, and L. Freedman, "Using a GIS to model interventions to strengthen the emergency referral system for maternal and newborn health in Ethiopia," International Journal of Gynecology and Obstetrics, vol. 115, pp. 300-309, 2011.
[4] E. C. Fradelos, I. V. Papathanasiou, D. Mitsi, K. Tsaras, C. F. Kleisiaris, and L. Kourkouta, 2014, "Health based geographic information system (GIS) and their applications," Review / ACTA Inform Med., vol. 22, no. 6, pp. 402-405, 2014.

[5] S. Khodambashi, "Business process re-engineering application in healthcare in a relation to health information systems," in Proc. International Conference on Health and Social Care Information Systems and Technologies. Procedia Technology, 2013, pp. 949-957.

[6] A. K. Jha, C. M. D. Roches et al., "Use of electronic health records in U.S. hospitals," The New England Journal of Medicine, vol. 360, no. 16, pp. 1628-1638, 2010.

[7] M. Ngafeeson, Healthcare Information Systems: Opportunities and Challenges, vol. 14, pp. 258-267, 2014.

[8] T. S. Babicheva, "The use of queuing theory at research and optimization of traffic on the signal-controlled road intersections," Procedia Computer Science, vol. 55, pp. 469-478, 2015.

[9] A. Bressan, S. Canic, M. Garavello, M. Herty, and B. Piccoli, 2014, "Flows on networks: recent results and perspectives," EMS Surveys in Mathematical Sciences, vol. 1, pp. 47-111, 2014

[10] A. Faro and D. Giordano, "Algorithms to find shortest and alternative paths in free flow and congested traffic regimes," Transportation Research Part C: Emerging Technologies, vol. 73, pp. 1-29, 2016.

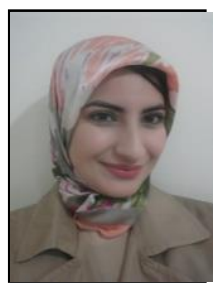

Ibtissam Khalfaoui was born in Oujda, Morocco. She graduated from Mohammed V International Academy of Civil Aviation of Casablanca in 2012, with an engineering degree in industry and production. She is currently preparing her thesis in engineering, management and optimization of Systems domain in Mohammadia School of Engineers of Rabat, Morocco.

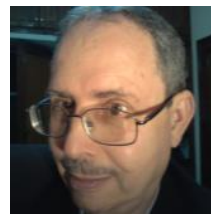

Amar Hammouche was born in Taza, Morocco. He holds a mechanical engineering degree from Mohammadia School of Engineers, Med V University, Rabat, Morocco, an $\mathrm{Ms}$ and a $\mathrm{PhD}$ in industrial engineering from Auburn University, USA. His main interests are in digital and intelligent manufacturing systems; systems design, modelisation and optimization; and production and quality planning and control. 